\section{Calculation of the Internal Surface of a Lung}

THE following investigation arose out of work carried on in the Pathology Department of King's College, University of Durham, by Prof. J. B. Duguid and his colleagues, for which an estimate was needed of the respiratory surface area within a given volume of lung.

Tomkeieff ${ }^{1}$ showed that, in a convex threedimensional figure, the average linear intercept is equal to four times the volume divided by the surface area ; that is,

$$
\bar{l}=4 \times \frac{\text { Volume }}{\text { Surface area }} .
$$

If a number of similar such bodies, each of volume $v$ and surface area $A$, are enclosed at random in a unit volume $(V)$ and this volume is traversed by a number of lines of total length $T$, if also $L$ is the total length of these lines which is interior to the convex bodies, and $N$ is the number of intercepts, then

$$
\frac{L}{\bar{T}} \rightarrow \frac{\text { Volume of convex bodies }}{\text { Unit volume }}=\frac{L}{4 N V} \times \Sigma(A),
$$

and in the limit

$$
\frac{\Sigma(A)}{V}=4 \frac{N}{T} .
$$

Therefore, in the limit, the surface area per unit volume is equal to four times the number of intercepts divided by the total length of traverse.

It can further be shown that there is no necessity for imposing the restriction that the bodies should be similar to one another, or that they should be entirely enclosed in the unit volume. It is necessary, however, to postulate that the bodies should be randomly disposed towards the lines of traverse.

In the case of the internal surface area of a sample of a lung, the lung can be embedded in gelatine without shrinkage or distortion and cut in thin sections. These sections can then be scanned under a microscope, and all that is necessary to obtain the internal surface area is to count the number of intersections with cell walls that are made by the line of scanning and divide this number by two to give the number of intercepts. This scanning and counting can, in fact, be done electronically.

\section{H. CAMPBell}

King's College,

University of Durham,

Newcastle upon Tyne.

$$
\text { April } 3 .
$$

1 Nature, 155, 24 (1945); correction, 1. 107.

\section{Hæmolytic and Surface Activity of Sodium Taurocholate}

IT is generally accepted that sodium taurocholate is a good lytic agent for erythrocytes, and a great number of reports on hæmolysis by this substance have been published ${ }^{1}$. Abnormal sigmoid timedilution curves are sometimes reported for taurocholate lysis, and frequent reference is made to the instability of the taurocholate solutions used by these authors. One report ${ }^{2}$ states qualitatively that a synthetic specimen of taurocholate was "moderately lytic", whereas a specimen prepared from natural sources was "strongly lytic". Two other

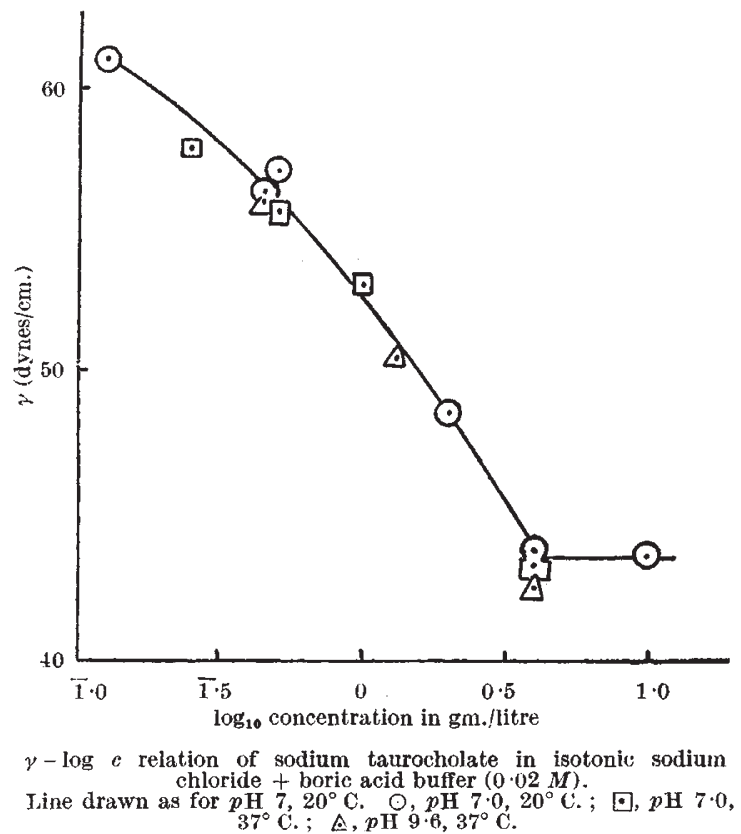

reports ${ }^{3}$ also give a much lower hæmolytic activity for taurocholate than that generally accepted.

Through the generosity of Dr. Stenhagen, we have recently obtained a very pure specimen of sodium taurocholate, which has been examined for its hæmolytic and surface activity. Hæmolysis tests were made at $20^{\circ} \mathrm{C}$. with a 5 per cent suspension of thrice-washed human erythrocytes $(A B, R h+)$ in $0 \cdot 85$ per cent sodium chloride buffered with $0.02 M$ boric acid, with borax added to $p \mathbf{H} 7 \cdot 0$. No lysis at all was observed overnight in concentrations of taurocholate up to $2.0 \mathrm{gm}$./lit. A solution of $11 \cdot 0$ $\mathrm{gm}$./lit. effected complete lysis in approximately $30 \mathrm{hr}$., and one of $19.8 \mathrm{gm}$./lit. in $90 \mathrm{~min}$. The lytic threshold for pure sodium taurocholate is therefore about $20 \mathrm{gm}$./lit. at $20^{\circ} \mathrm{C}$. The values for human cells given in the papers already quoted are in the range of $0 \cdot 10-0 \cdot 15 \mathrm{gm}$./lit. for the lytic threshold. It is concluded that the previously accepted high Iytic activity of sodium taurocholate is to be ascribed to the presence of impurities, perhaps long-chain fatty acids. The low activity of pure taurocholate has been confirmed by further experiments showing that a sample of natural taurocholate purified chromatographically and generously supplied by Dr. Pover has no lytic activity up to $3 \mathrm{gm}$./lit.

Determinations of surface tension were made at $p \mathrm{H} 7 \cdot 0$ and $p \mathrm{H} 9.5$, and at $20^{\circ} \mathrm{C}$. and $37^{\circ} \mathrm{C}$. on solutions of Dr. Stenhagen's sample in the isotonic saline boric acid buffers described above. The Harkins drop-volume method was used in these determinations, with a glass-tipped tuberculin syringe operated by a micrometer screw. No decomposition of the test solutions was observed at room temperature, and in agreement with the opinion of Hutchinson $^{4}$, no surface tension ageing of the bile salt solutions was observed with this pure specimen. The results are given on a logarithmic concentration scale in the accompanying graph. The micelle point of the sodium taurocholate is $4.1 \mathrm{gm}$./lit. $\left(7.4 \times 10^{-3} \mathrm{M}\right)$ in this medium and the maximum micellar surface pressure is $29 \cdot 3$ dynes. A full report will be published elsewhere to show that solutions of surface-active 